\title{
ESTRATEGIAS PARA LA DIVULGACIÓN DE LA CONTAMINACIÓN SÓNICA Y VISUAL EN EL PASEO CIENCIAS DE MARACAIBO, ESTADO ZULIA
}

\author{
Strategies for release of pollution visual and sonica walk in science of \\ Maracaibo, Zulia state
}

\section{César Morales*}

\section{RESUMEN}

Se presenta una investigación no experimental, descriptiva y de campo con recolección de datos transversales, cuyos objetivos fueron caracterizar la contaminación visual y sónica y aplicar estrategias de divulgación de dichos contaminantes físicos en el paseo ciencias de Maracaibo. Para el desarrollo de este trabajo se consideraron las propuestas de Fuentes y Arguello (2015), Méndez, (2013), Canelones (2007), Guijarro et al., (2015), Alfie y Salinas (2017), Espejo (2014) y Ayala (2017). Se usaron técnicas de observación directa e indirecta con sus respectivos instrumentos previamente elaborados. Para la observación directa se construyó una matriz que permitió identificar las fuentes de contaminación sónica y visual del área y para la observación indirecta se realizó una entrevista con preguntas abiertas. Los resultados obtenidos demuestran que el paseo ciencias por ser una zona comercial dinámica posee abundante contaminación visual y sónica en casi todos los elementos que componen sus fuentes. La música a alto volumen, los electrodomésticos, las sirenas y los autobuses son los contaminantes sónicos más destacados, mientras que los basurales improvisados, vallas publicitarias, los toldos y kioscos fueron los más nombrados como contaminantes visuales. Así mismo se indica que la elevada presencia de estos contaminantes altera los estados anímicos, físicos y las relaciones interpersonales de los ciudadanos que a diario se exponen a ellos y que el daño es proporcional al periodo de exposición. Es por ello que los entes municipales deben intervenir este espacio y buscar soluciones a la problemática en cuestión. El presente estudio demostró que existe una actitud muy pasiva por parte de la muestra para participar en programas que busquen corregir la situación. Finalmente se comprobó que son pocas las estrategias que se pueden aplicar para concientizar a los transeúntes y vendedores de este espacio comercial.

\footnotetext{
* Licenciado en Educación Mención Biología. Especialista en Educación Ambiental. Docente adjunto en U.E Nacional Dr. Máximo Arteaga Pérez. Investigador P.E.I.I. https://scholar.google.es/citations?user=ayEJ2ogAAAAJ\&hl=es http://orcid.org/0000-0002-5635-679X Maracaibo, Venezuela. Correo electrónico: cesarambienteluz@ planetmail.com
} 
Estrategias para la divulgación de la contaminación sónica y visual en el Paseo Ciencias de Maracaibo, estado Zulia

Palabras clave: contaminación sónica, contaminación visual, transeúntes, vendedores informales.

\begin{abstract}
An investigation is presented no experimental, descriptive field with data collection which aimed transverse characterized visual and noise pollution and implement strategies disclosure contaminants such physical at Maracaibo's Paseo Ciencias. For the developing of these work it's consider the proposal of Fuentes and Arguello (2015), Méndez, (2013), Canelones (2007), Guijarro et al., (2015), Alfie and Salinas (2017), Espejo (2014) and Ayala (2017). Direct and indirect observational techniques were used with the respective previously elaborated instruments. About direct observation, a matrix was built in order to identify the sources of sonic and visual pollution in the area. For the porpuse of indirect observation, a survey with open-ended questions was conducted. The results obtained demonstrate that Paseo Ciencias, as it's a commercial and dynamic zone, possesses a huge amount of sonic and visual pollution in almost every element that composes its sources. High volume music, electrodomestics, sirens and buses are the main sonic pollutants, whereas dumpsites, billboards, canopies, and kiosks are the major visual pollutants. Moreover, it is stated that the soaring presence of these pollutants disrupt the animic and physical states, and interpersonal relationships between citizens daily exposed to them and that the damage is proportional to the time of exposure. For these reasons, municipal entities must intervene in this area and search for solutions in order to solve the identified problems. This research demonstrates the sample has a very passive attitude towards participation in programs searching for a way to amend the situation. Finally, this research proved there are few suitable strategies to raise public awareness in this commercial site.
\end{abstract}

Keywords: sonic pollution, visual pollution, bystanders, sellers.

\title{
Introducción
}

Desde que el ser humano apareció en el planeta siempre ha buscado satisfacer sus necesidades básicas obteniendo recursos del ambiente (Guevara et al., 2015). En dicha acción han sido pocas las veces en las que se mide el daño que se le ocasiona a la naturaleza. De hecho mientras la especie humana busca adaptarse y adaptar el medio ambiente a sus necesidades se producen alteraciones que en muchos casos terminan 


\section{César Morales}

Telos Vol. 21, No. 2 (2019). 347-371

convirtiéndose en contaminación (Morales, 2016). De esta última se han logrado identificar diversas clases de contaminantes dentro de los cuales la contaminación sónica y visual adquieren un especial interés pues se están acentuado cada vez más en la ciudades y mayormente en las zonas comerciales (Méndez, 2013).

Así pues investigadores como Fuentes y Arguello (2015), Méndez, (2013), Canelones (2007), Guijarro et al., (2015), Alfie y Salinas (2017), Espejo (2014) y Ayala (2017), han identificado diversas fuentes de contaminación sónica y visual en la ciudades entre las cuales mencionan las antenas, el cableado aéreo, los basurales improvisados, los toldos de vendedores informales, las pintadas y grafitis, arboles mal colocados, sonidos de bocinas, gritos de vendedores, los sistemas de aceleración de autos, entre otros. Y en este mismo orden de ideas González y Fernández (2014) Informan que aunque estos contaminantes no generan gran daño al medio ambiente su efecto casi inmediato se da en los humanos quienes al exponerse a estos contaminantes suelen padecer una serie de efectos fisiológicos, psicológicos y sociales que hacen cada vez peor su calidad de vida.

En relación al área donde se realizó el presente estudio, autores como Muñoz (2012) informan que la contaminación acústica está cada vez más concentrada y Canelones (2007), demostró que los niveles sónicos en el casco histórico de Maracaibo están muy por encima de los niveles permitidos.

Ahora bien, Maracaibo es uno de los municipios más importantes de Venezuela (Contraloría General de la República, 2016); y su casco histórico es una zona de interés turístico a tal punto que sobre el reposan cuatro decretos patrimoniales: en el ámbito local como "patrimonio histórico arquitectónico" (Alcaldía del municipio Maracaibo, 1992); a nivel estadal, "remodélese y consérvese al barrio Santa Lucia" (Asamblea Legislativa del estado Zulia, 1976); a nivel nacional, "área de valor histórico" (Congreso de la República de Venezuela, 1994), y recientemente declarado "zona de interés turístico" (Asamblea Nacional, 2013). 
Estrategias para la divulgación de la contaminación sónica y visual en el Paseo Ciencias de Maracaibo, estado Zulia

En dicho espacio geográfico se encuentran la mayor cantidad de atractivos arquitectónicos y una gran variedad de comercios. Lo anteriormente expuesto, es un causal para que los turistas visiten el centro de dicho municipio. De hecho Camacaro y López (2015), informan que la visita al casco histórico es una de las 5 cosas que más hacen los turistas, y si los vendedores que laboran en dicha área han de continuar con sus acciones contaminantes el casco central se ira convirtiendo en una zona que ha de generar descontento en las personas que visitan diariamente ese lugar.

Por lo anteriormente expuesto se realizó la siguiente investigación, la cual tuvo como objetivo caracterizar la contaminación visual y sónica y aplicar estrategias de divulgación de dichos contaminantes físicos en el paseo ciencias de Maracaibo (Venezuela). La misma se llevó a cabo entre febrero del 2015 a mayo del 2016.

El presente estudio se justifica desde varios puntos de vista especialmente el ambiental pues identifica las fuentes iniciales de la contaminación sónica y visual en un espacio muy concurrido por los ciudadanos del municipio y propone nuevas líneas de acción afín de aplicar medidas mitigantes y correctivas en la área de estudio.

\section{Metodología}

La presente investigación se cataloga como factible pues permite la elaboración de una propuesta de un modelo operativo viable, o una solución posible, cuyo propósito es satisfacer una necesidad o solucionar un problema (Mosqueda, 2012). También es de campo, no experimental y del tipo transversal; llevada a cabo con una muestra representativa e informal de la población del paseo ciencias del casco histórico de Maracaibo (Venezuela).

Se realizaron dos tipos de muestreos según la necesidad del estudio. El primero fue un muestreo intencionado y se aplicó para aquellos individuos que trabajan en las inmediaciones del paseo ciencias y que por sus actividades son generadores de contaminación sónica y visual. El segundo muestreo es del tipo no probabilístico casual, 


\section{César Morales}

Telos Vol. 21, No. 2 (2019). 347-371

ya que los individuos se eligieron sin ningún juicio previo. Este último se utilizó para los ciudadanos que a diario transitan por el área de estudio y que por su naturaleza ameritan un tipo diferente de muestreo. Se escogieron 40 personas a las cuales se les realizo la entrevista la cual duro entre 5 y 15 minutos.

La técnica de recolección de datos usada fue la observación directa e indirecta. Para la técnica de la observación directa se usó como instrumento una lista de cotejo elaborada por el investigador siguiendo las propuestas de Fuentes y Arguello (2015), Espejo (2014), Méndez, (2013), Guijarro et al. (2015), Ayala (2017), Alfie y Salinas (2017) y Canelones (2007). Dicho instrumento facilitó el registro de los datos observados en relación a la contaminación sónica y visual. Esta lista de cotejo ofrece como atributos el contener la mayor cantidad posible de detalles del problema, considerando para ello la variable, sus dimensiones e indicadores. Y para la técnica de observación indirecta se realizó una entrevista abierta.

La entrevista se aplicó en el campo a las 40 personas tomadas al azar que caminaban por el espacio de estudio y a vendedores del área. La misma fue grabada en audio con un teléfono celular. Dicha entrevista estuvo conformada por 9 preguntas guía (Tabla 4) distribuidas equitativamente entre las 5 dimensiones que representa la variable contaminación sónica y visual (Tabla 1). Luego del trabajo de campo se procedió a la transcripción de los resultados de las entrevistas y a la elaboración de un memo tal y como sugieren Borda et al. (2017). Posteriormente, se sistematizaron, de forma descriptiva e interpretativa, las respuestas obtenidas de los informantes y se analizan y discuten organizadas en las dimensiones fuentes de contaminación sónica, consecuencias de la contaminación sónica, fuentes de contaminación visual, consecuencias de la contaminación visual y grado de participación.

\section{TABLA 1.}

\section{MAPA DE VARIABLE}

\begin{tabular}{|c|c|c|c|c|}
\hline \multicolumn{1}{|c|}{ Variable } & Dimensión & & \multicolumn{1}{c|}{ Indicadores } & Ítem \\
\hline Contaminación & Fuentes de & $>$ & Fijas. & $>1$ \\
sónica y visual & contaminación sónica & $>$ & Móviles. & $>2$ \\
\hline
\end{tabular}


Estrategias para la divulgación de la contaminación sónica y visual en el Paseo Ciencias de Maracaibo, estado Zulia

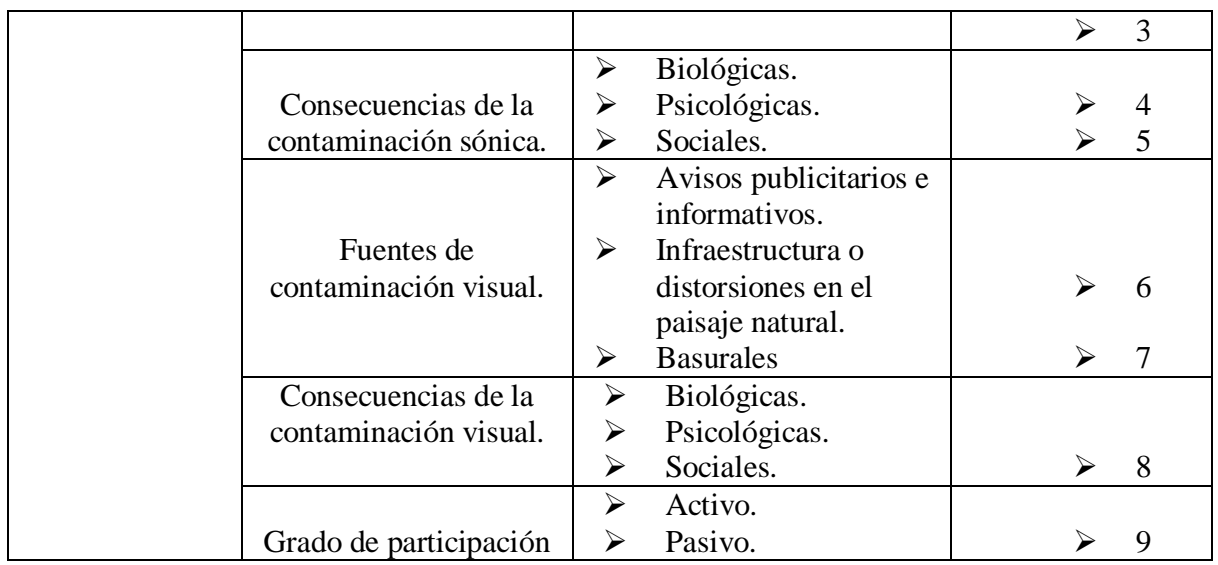

Fuente: Elaboración propia.

En base a los resultados obtenidos de los instrumentos de recolección de datos, se seleccionaron contenidos procedimentales, conceptuales y actitudinales relacionados con la contaminación sónica y visual que deberían mejorarse o modificarse en el área de estudio, afín de promover actitudes mitigantes con respecto a los contaminantes físicos en cuestión.

Para ello, se diseñó una serie de estrategias pedagógicas las cuales se estructuraron en tres bloques de actividades (tabla 5): inicio, desarrollo y evaluación de cierre. Durante la fase de inicio se buscó activar los conocimientos previos haciendo uso de la técnica de la pregunta y de un "sabias que" tal como fue aplicado en la experiencia sistematizada por Morales (2016). Asimismo se realizó la entrega de dípticos informativos; en la fase de desarrollo se aplicaron charlas informativas individuales a comerciantes y transeúntes del paseo ciencias. Y En la fase del cierre se realizó una retroalimentación y un análisis descriptivo del proceso de aplicación de la propuesta. 
César Morales

Telos Vol. 21, No. 2 (2019). 347-371

\section{Resultados}

Los resultados de la observación directa se colectaron con la ayuda de una matriz de elaboración propia (tabla 2). En dicho instrumento, aplicado a las fuentes de contaminación visual, se evidencia que del 100\% de los contaminantes tomados en cuenta para verificar su presencia en el área de estudio, el 75\% están presentes y solo el $25 \%$ está ausente.

\section{TABLA 2. RESULTADOS DE LA CONTAMINACIÓN VISUAL POR OBSERVACIÓN DIRECTA}

\begin{tabular}{|c|c|c|}
\hline & PRESENTE & AUSENTE \\
\hline Carteles publicitarios & $\mathrm{X}$ & \\
\hline Grafitis & $\mathrm{X}$ & $\mathrm{X}$ \\
\hline Pintadas & $\mathrm{X}$ & \\
\hline Antenas & $\mathrm{X}$ & \\
\hline Tendido de cables & $\mathrm{X}$ & \\
\hline Postes y Chimeneas & $\mathrm{X}$ & $\mathrm{X}$ \\
\hline Basurales & & \\
\hline Plantas mal colocadas & &
\end{tabular}

Fuente: Elaboración propia

Para la observación directa de las fuentes de contaminación sónica también se usó una matriz (Tabla 3), donde se agruparon los contaminantes en cuestión y se evidenció que del 100\% de las manifestaciones de las fuentes de contaminación sónica, en el área de estudio se encuentran un 58,8\% de los mismos.

\section{TABLA 3. RESULTADOS DE LA CONTAMINACIÓN SONICA POR OBSERVACIÓN DIRECTA}

\begin{tabular}{|c|c|c|c|}
\hline & & PRESENTE & AUSENTE \\
\hline \multirow{8}{*}{$\begin{array}{l}\text { Contaminantes } \\
\text { Móviles }\end{array}$} & Producidos por sistemas de aceleración. & $\mathrm{X}$ & \\
\hline & Producidos por escapes modificados. & $\mathrm{X}$ & \\
\hline & Producidos por toques de bocinas. & $\mathrm{X}$ & \\
\hline & Equipos de sonido a alto volumen. & $\mathrm{X}$ & \\
\hline & Ruidos de la carrocería. & & $\mathrm{X}$ \\
\hline & Ruidos de sirenas de autos permisibles. & $\mathrm{X}$ & \\
\hline & Alarmas contra robos & $\mathrm{X}$ & \\
\hline & Ruido de aviones. & & $\mathrm{X}$ \\
\hline
\end{tabular}


Estrategias para la divulgación de la contaminación sónica y visual en el Paseo Ciencias de Maracaibo, estado Zulia

\begin{tabular}{|c|l|c|c|}
\hline \multirow{5}{*}{$\begin{array}{c}\text { Contaminantes } \\
\text { fijos }\end{array}$} & $\begin{array}{l}\text { Relacionadas a obras públicas y } \\
\text { lonstrucciones. }\end{array}$ & & $\mathrm{X}$ \\
\cline { 2 - 4 } & $\begin{array}{l}\text { Relacionadas a servicios de limpieza y } \\
\text { recolección de residuos sólidos. }\end{array}$ & & $\mathrm{X}$ \\
\cline { 2 - 4 } & Alarmas de locales comerciales. & & $\mathrm{X}$ \\
\cline { 2 - 4 } & $\begin{array}{l}\text { Relacionadas a actividades lúdicas y } \\
\text { recreativas. }\end{array}$ & & $\mathrm{X}$ \\
\cline { 2 - 4 } & Ruido de artefactos electrodomésticos & $\mathrm{X}$ & \\
\cline { 2 - 4 } & Gritos de niños jugando & $\mathrm{X}$ & \\
\cline { 2 - 4 } & Conversaciones o peleas en la calle & $\mathrm{X}$ & $\mathrm{X}$ \\
\cline { 2 - 4 } & $\begin{array}{l}\text { Televisión o radios en viviendas a alto } \\
\text { volumen }\end{array}$ & & \\
\cline { 2 - 4 } & Ruido producido por animales & & \\
\hline
\end{tabular}

Fuente: Elaboración propia

Para la observación indirecta se realizaron entrevistas a personas tomadas al azar que caminaban por el espacio de estudio y a vendedores del área del Paseo Ciencias. Los hallazgos generales de la entrevista se sistematizaron en una tabla (tabla 4), con el fin de hacer más didáctico los diversos aportes de la muestra.

TABLA 4.

\section{RESULTADOS DE LA CONTAMINACIÓN SONICA Y VISUAL POR OBSERVACIÓN INDIRECTA}

\begin{tabular}{|c|l|l|}
\hline \multirow{2}{*}{$\mathbf{1}$} & $\begin{array}{l}\text { Preguntas de acercamiento } \\
\text { alrededontaminación sónica en los la calle Paseo Ciencias? }\end{array}$ & $\begin{array}{l}\text { Hallazgos en la muestra } \\
\text { la contaminación sónica. Y que entre } \\
\text { semana (lunes a viernes) hay más } \\
\text { contaminación sónica que en los sábados y } \\
\text { domingos. }\end{array}$ \\
\hline $\mathbf{2}$ & $\begin{array}{l}\text { ¿Puedes señalar o decir algunos } \\
\text { ejemplos de contaminación sónica fija? }\end{array}$ & $\begin{array}{l}\text { Señalan a los equipos de sonido a alto } \\
\text { volumen como contaminación sónica por } \\
\text { fuentes fija y a los gritos de personas en el } \\
\text { área, los cuales están representados casi en } \\
\text { sutotalidad por vendedores informales }\end{array}$ \\
\hline $\mathbf{3}$ & $\begin{array}{l}\text { ¿Puedes señalar o decir algunos } \\
\text { ejemplos de contaminación sónica } \\
\text { móvil? }\end{array}$ & $\begin{array}{l}\text { Los vehículos y autobuses son la principal } \\
\text { fuente de contaminante sónico móvil. }\end{array}$ \\
\hline $\mathbf{4}$ & $\begin{array}{l}\text { ¿Cuáles son las consecuencias de estar } \\
\text { expuesto a dicha contaminación? }\end{array}$ & $\begin{array}{l}\text { Reportan una mayor frecuencia de } \\
\text { malestares psicológicos (estrés, sentirse } \\
\text { agitadas, de mal humor e impotentes). } \\
\text { Consecuencias biológicas (cansancio, }\end{array}$ \\
\hline
\end{tabular}




\begin{tabular}{|c|c|c|}
\hline & & $\begin{array}{l}\text { efectos a nivel auditivo y cefaleas). Y } \\
\text { consecuencias sociales (pleitos y ofensas). }\end{array}$ \\
\hline 5 & $\begin{array}{l}\text { ¿Cómo te sientes después de salir de un } \\
\text { ambiente tan contaminado sónicamente? }\end{array}$ & $\begin{array}{l}\text { Experimentan cansancio, que aun después } \\
\text { de haber salido del ambiente ruidoso lo } \\
\text { sienten. }\end{array}$ \\
\hline 6 & $\begin{array}{l}\text { ¿Crees que hay contaminación visual en } \\
\text { los alrededores de dicha calle? }\end{array}$ & $\begin{array}{l}\text { El 83\% de las personas que participaron en } \\
\text { el estudio saben identificar la } \\
\text { contaminación visual y además afirman } \\
\text { que dicha contaminación está ampliamente } \\
\text { extendida en el paseo ciencias del casco } \\
\text { histórico }\end{array}$ \\
\hline 7 & $\begin{array}{l}\text { ¿Puedes señalar o decir algún ejemplo } \\
\text { de contaminación visual en el área? }\end{array}$ & $\begin{array}{l}\text { Los avisos publicitarios son el ejemplo de } \\
\text { contaminación visual más mencionado. } \\
\text { Seguido de los vendedores informales y los } \\
\text { basurales improvisados. }\end{array}$ \\
\hline 8 & $\begin{array}{l}\text { ¿Cuáles crees que sean las } \\
\text { consecuencias de estar expuesto a la } \\
\text { Contaminación visual en el área? }\end{array}$ & $\begin{array}{l}\text { Cansancio, como si su fuerza fuera de } \\
\text { alguna manera absorbida. Fatiga, } \\
\text { sentimientos de Repulsión, indignación, } \\
\text { depresión y decepción. }\end{array}$ \\
\hline 9 & $\begin{array}{l}\text { ¿Estarías dispuesto (a) participar en un } \\
\text { proyecto o actividad que busque } \\
\text { minimizar la presencia de la } \\
\text { contaminación sónica y visual en esta } \\
\text { área? }\end{array}$ & $\begin{array}{l}\text { Las personas tienen un grado de } \\
\text { participación altamente pasivo. }\end{array}$ \\
\hline
\end{tabular}

Fuente: Elaboración propia.

\section{Discusiones}

Fuentes de contaminación sónica (fija y móvil).

Los resultados demuestran primeramente que los informantes reconocen la presencia y los altos niveles de ruido en el área. Esto último, fue reportado por Canelones (2007), quien informó que es por causa del congestionamiento vehicular que se percibe altos niveles de aceleración del motor, activación de cornetas y sirenas sin control. De igual forma, el mismo investigador corrobora lo que informan los entrevistados: que en los días de feria y en navidad se percibe un incremento significativo en el ruido producto del aumento de los vendedores informales que se establecen en el casco histórico.

Todos los datos aportados por las entrevistas, son ratificados por el investigador en la matriz de observación directa donde no solo se informa la presencia 
Estrategias para la divulgación de la contaminación sónica y visual en el Paseo Ciencias de Maracaibo, estado Zulia

de contaminación sónica sino que también se identifican las fuentes de esta contaminación. Todo esto, como diría Canelones (2007), "Han hecho de ella (Maracaibo) una ciudad caótica, desordenada y contaminada" (p. 13).

Con respecto a las fuentes de contaminación sónica en el paseo ciencias, están representadas en su mayoría por fuentes fijas, siendo la más representativa la producida por los equipos de sonidos de los comerciantes del área. Estos equipos de sonido están presentes en la mayoría de los locales y comercios del espacio estudiado, y es que el paseo ciencias por ser una zona comercial tiene como característica la presencia de este tipo de contaminante (Muñoz, 2012). De igual forma Canelones (2007) informó que los ruidos de electrodomésticos en las zonas residenciales del casco histórico están categorizados como contaminantes sónicos fijos. Dicho malestar se observa que persiste desde la época hasta ahora.

Así mismo, los gritos afanados de los vendedores es una de las fuentes de contaminación por ruidos fijos que perciben los informantes con más intensidad, ya que fue el segundo más mencionado por la muestra. Estos vendedores están representados casi en su totalidad por el sector informal y vendedores ambulantes que gritan con afán los productos que ofrecen en sus kioscos. Estos gritos molestan a los transeúntes a tal punto que durante la entrevista no dejaban escuchar lo que se les preguntaba, es por ello que tal situación fue identificada por los entrevistados como contaminación sónica.

De modo idéntico, se observó que hay otros tipos de contaminantes sónicos fijos que no fueron mencionados por los entrevistados, pero que están presentes en el área. Estos fueron identificados con ayuda de la matriz de observación directa usada por el investigador. Entre estos están los Gritos de niños jugando; al respecto investigadores tales como Guijarro et al., (2016) y Muñoz (2012), determinaron que entre las fuentes de contaminación sónica fija, las de origen comunitario específicamente las de niños practicando actividades lúdicas, de ocio y recreativas, representan una de las 5 fuentes emisoras de ruido más generales de los urbanismos. De 


\section{César Morales}

Telos Vol. 21, No. 2 (2019). 347-371

igual manera se determinó que hay conversaciones o peleas en la calle y televisión o radios en viviendas a alto volumen.

Por otra parte, la segunda fuente de contaminación sónica identificada en el sitio de estudio es la referida a las fuentes móviles. Se encontró que la totalidad de los entrevistados informan que la contaminación por fuentes móviles en el área está representada por los autos y autobuses. Esto concuerda con lo expuesto por Alfie y Salina (2017), quienes aseveran que la contaminación por fuentes móviles está constituida por el tránsito de vehículos y motorizados.

Conviene subrayar que en dicho espacio geográfico concurre una gran cantidad de carros por puestos y autobuses de transporte público, además la gran mayoría de ese transporte terrestre que circula por el casco histórico de Maracaibo tienen su parada en esta área es por eso que ha de generarse una gran cantidad de ruidos contaminantes. A esto último se le suma el ruido de las sirenas de las ambulancias del hospital Nuestra Señora de Chiquinquirá, el sonido de las bocinas de la gran cantidad de autos por puestos y demás medios de transporte publico quienes se ven inmersos en embotellamientos diarios.

No obstante el problema no termina allí pues para los transeúntes que suben al transporte público se encuentran con otra fuente de contaminación sónica móvil: La contaminación dentro del transporte público. Esto último es confirmado por Miyara y Sanguineti (1996), cuando dicen que "En los vehículos utilizados para el transporte urbano de pasajeros existe habitualmente un nivel de contaminación acústica bastante significativo, debido a varias fuentes de ruido y a las propiedades resonantes y acústicoconductoras de la carrocería" (p.2). De igual forma los mismos investigadores dicen que esta contaminación repercute negativamente en el estado de ánimo de los conductores de los autobuses y en la actitud frente a su trabajo y ante los pasajeros, y al perturbar la capacidad de percepción y consecuentemente los reflejos, reduce las condiciones de seguridad en que se presta el servicio. 
Estrategias para la divulgación de la contaminación sónica y visual en el Paseo Ciencias de Maracaibo, estado Zulia

Más aun, hay otros contaminantes sónicos móviles que fueron identificados por el investigador. Estos están presentes en el área de estudio y aunque no fueron mencionados por los informantes, lo cual sugiere un bajo conocimiento de la temática, están caracterizados por otros autores como contaminación por ruido (Miyara y Sanguineti, 1996). Estos son: La aceleración de los autobuses, los escapes modificados, las alarmas contra robo de autos, toques de bocinas y ruidos de sirenas de ambulancias.

Cabe destacar que en el paseo ciencias se encontró que la contaminación por fuentes móviles es la que menos perciben las personas. Esto se determinó al analizar las entrevistas pues los informantes reportan en mayor cantidad los contaminantes sónicos fijos (sonido de música a alto volumen, gritos de vendedores y conversaciones) y casi no mencionan a las fuentes móviles y en algunos casos informan que no está presente. Esto se puede deber, en primer lugar, a que el paseo ciencias por ser una zona comercial hay una gran cantidad de personas que caminan por las carreteras por lo cual los medios de transporte que circulan por dicha área se ven en la necesidad de llevar velocidades muy bajas. Esto es una gran salvedad pues no hay tanta frecuencia de contaminación por sistemas de aceleración y piques.

En segundo lugar, las personas no perciben con tanta intensidad la contaminación por ruido vehicular ya que están más cerca de los contaminantes fijos antes mencionados entonces puede ocurrir un encubrimiento de los ruidos por fuentes móviles. En este último caso se afirma que están allí, pero los otros ruidos no los dejan ser escuchados. Al respecto Canelones (2007) aclara que el encubrimiento se refiere a la disminución de la capacidad de percibir o entender un sonido en presencia de otro ruido.

Consecuencias de la contaminación sónica (Biológica, psicológica y social).

Con respecto a la segunda dimensión, las personas manifiestan experimentar una serie de efectos negativos tanto físicos, como psicológicos y sociales. A este 


\section{César Morales}

Telos Vol. 21, No. 2 (2019). 347-371

propósito afirma Muñoz (2012), que la contaminación por ruido es un mal "cuyos efectos nocivos sobre la salud físico-mental han sido demostrados en el área de la medicina (p.14). En el presente estudio se evidenció que las personas tienen efecto a nivel biológico, psicológico y social.

Las consecuencias a nivel biológico que sienten los transeúntes y vendedores del paseo ciencias son, cefalea, fatiga y sorderas momentáneas. Estos efectos biológicos que expresan las personas que transitan o trabajan en el área del paseo ciencias, pueden explicarse según lo expuesto por Rosales (2014) quien refiere que las personas expuestas constantemente a factores estresores del tipo sónico desencadenan en ellos estrés agudo y crónico que a su vez les generan "asma, urticaria, eccemas, diferentes tipos de dolor tales como dolor de cabeza, abdominal, síntomas gastrointestinales (indigestión, diarrea). Por el contrario, el estrés crónico genera trastornos metabólicos (obesidad, diabetes mellitus tipo 2) y trastornos cardiovasculares (hipertensión, infarto de miocardio)" (p.10).

El otro efecto biológico que dicen experimentar los entrevistados es un gran cansancio; y no es de extrañar que los transeúntes se sientan así pues en los ambientes ruidosos el aparato auditivo recibe un "bombardeo" constante de energía sonora que el cerebro y los nervios deben estar procesando constantemente, esto genera fatiga a nivel del oído pudiendo afectar el equilibrio y generando esa sensación de agotamiento físico (Canelones 2007). Ahora bien, con solo observar esta reacción ya se debe entender que se está comprometiendo seriamente la salud del visitante del casco histórico por causa de este contaminante físico y la falta de controles o estudios socio-ambientales que puedan mitigar el fenómeno.

Por otro lado, la consecuencia más mencionada es la que se produce a nivel psicológico, de esta forma las personas reportaron sentirse estresadas, de mal humor, irritadas, entre otros.

En la misma dimensión se encontró consecuencias sociales observándose pleitos y discusiones. Esto lo explica, Muñoz (2012) y Canelones (2007), y dicen que 
Estrategias para la divulgación de la contaminación sónica y visual en el Paseo Ciencias de Maracaibo, estado Zulia

los individuos pueden sentir una diversidad de emociones negativas cuando son expuestos al ruido comunitario, esto les hace sentirse insatisfechos, irritados, indefensos para dar soluciones al problema, ansiosas y agresivas. También Muñoz (2012) dice que las personas expuestas a contaminación sónica tienden a manifestar Irritabilidad exagerada que se puede convertir en hostilidad. Por ende, las relaciones interpersonales se deterioran rápidamente.

Finalmente, se consiguió que persisten las interferencias en la comunicación hablada (González y Fernández, 2014). De esto último afirma Canelones (2007) que "aunque los ruidos sean de muy baja intensidad impiden una comunicación efectiva y la elaboración de ideas" (p.29). De igual forma, sigue refiriendo el mismo autor que “También son afectados los niños, debido a que la etapa inicial de la adquisición del lenguaje debe realizarse en una atmósfera sin ruidos para que no afecte su comprensión en los conocimientos aprendidos" (p. 29).

Fuentes de contaminación visual (Avisos publicitarios e informativos, Infraestructura o distorsiones en el paisaje natural y Basurales).

Para el sexto ítem las personas concuerdan en que en el paseo ciencias del centro de Maracaibo existen niveles alarmantes de contaminación visual. Autores como Ayala (2017) afirma que este tipo de contaminación es característica de estos entornos urbanos y su presencia en tales áreas se debe principalmente a actividades humanas.

Entre las fuentes de contaminación visual establecidas en la literatura, se encontró que en el paseo ciencias los toldos y puestos improvisados son mencionados como uno de los contaminantes visuales que más afean el medio. Y es que estos puestos de los comerciantes degradan la estética ambiental de la zona a niveles muy bajos, a opinión del investigador, esto se debe a dos factores: en primer lugar los techos (en su mayoría de zinc oxidado) generan discordancia con la arquitectura del lugar; en segundo lugar porque desvirtúan y opacan la visibilidad que pueda tener el visitante del casco 


\section{César Morales}

Telos Vol. 21, No. 2 (2019). 347-371

histórico. Todo esto genera, según Méndez (2013) pérdida de valores escénicos y distorsiones en el paisaje.

También se menciona la mala disposición de los desechos sólidos, los avisos y vallas publicitarias como principales generadores de contaminación visual. En lo que se refiere a los basurales improvisados, se observa que en el área hay una mala disposición de desechos sólidos pues vendedores y transeúntes arrojan toda clase de desechos en el suelo. Al respecto Méndez (2013) menciona que el 29\% de la contaminación visual es generada por basura mal dispuesta. "En su mayoría las personas asocian su presencia a malos olores, a que afean el lugar y se percibe contaminación; a otros les produce sensación de pánico, repulsión, asco" (Méndez, 2013 p. 11). Cabe destacar que este último es el contaminante visual que ameritó más artículos en la prensa local en lo que va de año.

Ahora bien, hay otra cantidad significativa de contaminantes visuales que las personas no mencionaron lo cual demuestra poco conocimiento de lo que es la contaminación visual. Entre estos están las pintadas; las cuales están en las paredes de las edificaciones en forma de propaganda política. Este mensaje político más que una publicidad es un contaminante visual que puede afectar al 30\% de la población (Méndez, 2013). De igual forma, refiere la misma autora que las pintadas "resultan molestos porque deterioran la propiedad pública y privada degradando el entorno, dan la sensación de inseguridad, vandalismo, impotencia, desagrado, afean la ciudad, dan la impresión de descuido y falta de educación de la población que los realiza" (p.10).

Cabe destacar que aunque el cableado aéreo no fue mencionado por los entrevistados no es indicativo de su total ausencia pues en observación del investigador se corroboro la abundancia de este contaminante, pues se observaron tomas ilegales de electricidad a tal magnitud que los postes se ven insuficiente para sostener tal densidad de cableado. En referencia a este contaminante visual Méndez (2013), dice que "genera principalmente sensación de peligro e inseguridad. Los sujetos encuestados afirman que 
Estrategias para la divulgación de la contaminación sónica y visual en el Paseo Ciencias de Maracaibo, estado Zulia

deslucen el entorno, dan la sensación de caos y desorden, obstaculizan las visuales del paisaje y producen incomodidad" (p. 11).

La contaminación visual por árboles mal colocados (Fuentes y arguello, 2015) está totalmente ausente en esta calle. Las antenas (de TV, las Parabólicas y de telecomunicaciones) en el área están muy reducidas en número. Sin embargo son elementos que autores como Sáez (2011) sugieren que son contaminantes visuales porque van contra la conservación ecológica en las zonas de conjuntos monumentales y su presencia es indicativo de descuido de la estética del paisaje.

Consecuencias de la contaminación visual (biológica, psicológica y social).

En la dimensión de las consecuencias se evidencio que existen efectos biológicos que reportan los transeúntes expuestos a la contaminación visual. Entre estos se enumeran la fatiga y el cansancio. En opinión del investigador, la fatiga se debe principalmente a los estímulos visuales generados por la acumulación de basura que hay en el sitio especialmente cuando de su presencia en las aceras se trata, porque interfieren con la circulación de las personas. De igual forma, el olor putrefacto emitido por los basurales improvisados colabora directamente con la sensación de fatiga que inicia en la vista del transeúnte.

En cuanto a la sensación de cansancio que se encontró en la muestra esta ha sido reportada por Méndez (2013), quien explica que este se da por un agotamiento en la vista por percibir el ambiente con tanta falta de armonía y con un exceso de información comercial. Esto último es casi inevitable pues el paseo ciencias es una zona comercial muy dinámica y con un gran cantidad de avisos, vallas publicitarias y con una excesiva cantidad de vendedores informales.

De modo accesorio, hay otras consecuencias físicas que les puede ocurrir a los individuos que se exponen a la contaminación visual. Espejo (2014), dice que se produce una sobre estimulación visual agresiva y menciona el estrés visual como principal efecto a nivel corporal. Las consecuencias también se hacen sentir en el tejido 


\section{César Morales}

Telos Vol. 21, No. 2 (2019). 347-371

nervioso, esto se debe a que cuando el cerebro humano recibe más información visual de la que es capaz de procesar, produce un estado de tensiones en el sistema nervioso central (S.N.C) con efectos en la salud.

Por su parte, las consecuencias psicológicas que expresan padecer los transeúntes y vendedores son repulsión, estrés, indignación, depresión y decepción. Estos últimos malestares psicológicos tienen relación directa con las respuestas evaluativas y afectivas de las personas ante el paisaje contaminado visualmente. Estas pueden ser algunas razones por las cuales los lugares "feos" afectan el sentido de comodidad y por ende se siente menos conexión o responsabilidad con los mismos (Fuentes y Arguello, 2015).

A su vez, hay otras consecuencias psicológicas que pueden experimentar estas personas según los autores Méndez (2013), Fuentes y arguello (2015) y Espejo (2014), se produce confusión, ansiedad, falta de atención, fatiga cognoscitiva, disgusto, aburrimiento, irritabilidad, agresividad, mal humor, sensaciones de inseguridad y bajo rendimiento académico y laboral.

Para el punto de vista social, se observa que la sobre estimulación a la cual se ven expuestas las personas por efecto de las fuentes de contaminación visual son: pérdida de valores escénicos, falta de sentido de pertenencia, irresponsabilidad a la hora de disponer los desechos sólidos, poca colaboración de las personas para recuperar el área y desordenes arquitectónicos. Estos efectos han sido reportados por Méndez (2013) y Espejo (2014), quienes estudiaron como el aspecto de las edificaciones condiciona las respuestas afectivas y evaluativas de las personas. Y explica que un lugar muy contaminado disminuye la conexión y la empatía que sienten las personas hacia el sitio. En otras palabras, las personas se sienten incomodas, desconectadas y sienten que no tienen responsabilidad alguna con respecto al espacio evaluado. Esto, aunado a la falta de tiempo y calor, es la razón por la cual intentar que todos participen en actividades de recuperación de la estética del paseo ciencias se hace sumamente difícil.

Grado de participación (activo y pasivo) 
Estrategias para la divulgación de la contaminación sónica y visual en el Paseo Ciencias de Maracaibo, estado Zulia

La dimensión grado de participación de las personas se resume en una negativa del $90 \%$ de los individuos de participar en programas de mejoramiento ambiental. Se afirma entonces que las personas que laboran y circulan por el paseo ciencias, en el momento que fue realizado este estudio, son casi en su totalidad pasivas. Este grado de participación tiene mucha relación con la contaminación visual del área y con la falta de responsabilidad que sienten las personas. Muchos creen que rescatar o mantener el área de estudio limpia es tarea de las autoridades gubernamentales.

Una de las razones de esta negativa para participar en planes de mejoramiento sónico y visual es expuesta por Méndez (2013) quien explica que un lugar feo y degradado ambientalmente induce en las personas una sensación de incomodidad y por ende se sentirán menos conectadas o responsables de mantener limpio dicho lugar.

En síntesis: la contaminación sónica y visual en la zona urbana y comercial conocida como el paseo ciencias, está ampliamente distribuida en todas sus fuentes. Aunque muchas personas que circulan por esta concurrida calle no reconocen a la totalidad de los contaminantes sónicos y visuales, lo que denota un escaso conocimiento de la variable en estudio, la observación directa del investigador identificó una elevada cantidad de estos contaminantes. $\mathrm{Y}$ es que estos contaminantes físicos se han hecho comunes y característicos de zonas urbanas y con elevada actividades comerciales como lo es el paseo ciencias.

Los datos de la variable indican que el área de estudio y el resto del casco histórico ha dejado de ser una zona atractiva, pues aseguran los visitantes entrevistados que ir a ese sector les genera repulsión, indignación y hasta los deprime; todo esto por el incremento significativo de los contaminantes estudiados.

Finalmente, se tiene como ventaja que estos contaminantes no tienen consecuencias sobre los factores abióticos (Muñoz, 2012), son fácilmente detectables y una vez que se destruye la fuente de inmediato desaparecen del ambiente sin dejar residuos. 


\section{CONCIENCIANDO LA COMUNIDAD DEL PASEO CIENCIAS PARA UN AMBIENTE LIBRE DE CONTAMINACIÓN SONICA Y VISUAL}

Propuesta de acción educativa para aminorar los efectos de la contaminación sónica y visual.

Esta propuesta surge de la necesidad de llevar a la acción actividades educativas y ambientales que logren llamar la atención de los involucrados y generar en ellos un cambio de actitud en pro del ambiente. Según los resultados obtenidos en la presente investigación se evidencia una necesidad de realizar actividades en el área del paseo ciencias para que los involucrados tengan mayor conciencia sobre la contaminación visual y sónica y puedan colaborar en disminuir las presencias de las fuentes de los contaminantes en cuestión.

Se considera que la propuesta que se plantea tiene relevancia social pues puede servir de guía y referencia para posteriores trabajos de educación ambiental en el área. De igual forma que en una futura intervención del casco histórico sea acogido el presente estudio y que sea aplicado en toda la extensión del espacio donde se aplicó el estudio. Así mismo, se plantea como misión que la siguiente propuesta pueda generar conocimientos y actitudes a beneficio del ambiente en transeúntes y vendedores del paseo ciencias y que en interacciones directas con el investigador las personas consideren cambiar sus hábitos. La siguiente propuesta tiene la finalidad de aplicar estrategias de divulgación de la contaminación sónica y visual en el paseo ciencias del casco histórico de Maracaibo en Venezuela.

En evaluaciones previas de la propuesta se puede mencionar sus fortalezas y debilidades. Entre las fortalezas se encuentran que el área es de libre entrada y se puede aplicar la charla a cualquier hora del día sin necesidad de permisología de ningún ente. Por otro lado, se están usando estrategias que fueron seleccionadas después de un riguroso estudio previamente desarrollado en las secciones anteriores, por lo cual, a 
Estrategias para la divulgación de la contaminación sónica y visual en el Paseo Ciencias de Maracaibo, estado Zulia

opinión del investigador, se tiene convicción de que estas estrategias son las más adecuadas para el escenario escogido. En cuanto a sus debilidades o amenazas se comprobó que la zona presenta cierto grado de inseguridad e insalubridad, y que por la naturaleza del trabajo de los vendedores o por la prisa que lleven los transeúntes el autor se vea interrumpido una y otra vez en el transcurso de las charlas. En la tabla número 4 se resume el plan de acción que se siguió durante la propuesta.

\section{TABLA 5. PLAN DE ACCIÓN}

\begin{tabular}{|c|c|c|c|c|c|c|}
\hline & Objetivos & Actividad & Recursos & $\begin{array}{c}\text { Población } \\
\text { Objetivo }\end{array}$ & $\begin{array}{c}\text { Indicador } \\
\text { de logro }\end{array}$ & $\begin{array}{l}\text { Medios de } \\
\text { verificación }\end{array}$ \\
\hline \multirow[b]{2}{*}{ 串 } & \multirow{2}{*}{$\begin{array}{l}\text { Divulgar } \\
\text { mediante } \\
\text { dípticos las } \\
\text { causas, } \\
\text { fuentes y } \\
\text { consecuencia } \\
\text { s de la } \\
\text { contaminació } \\
\text { n sónica y } \\
\text { visual. }\end{array}$} & \multirow{2}{*}{$\begin{array}{l}\text { Repartir } \\
\text { dípticos } \\
\text { informativos } \\
\text { a vendedores } \\
\text { y transeúntes. } \\
40 \text { como } \\
\text { mínimo }\end{array}$} & \multirow[t]{2}{*}{ Dípticos } & Transeúntes & \multirow[t]{2}{*}{$\begin{array}{l}\mathrm{Se} \\
\text { repartieron } \\
40 \text { dípticos }\end{array}$} & $\begin{array}{l}\text { Copia de los } \\
\text { dípticos }\end{array}$ \\
\hline & & & & Vendedores & & $\begin{array}{l}\text { Registro } \\
\text { fotográficos }\end{array}$ \\
\hline \multirow[b]{2}{*}{ 䏤 } & \multirow{2}{*}{$\begin{array}{l}\text { Aplicar } \\
\text { charlas } \\
\text { individuales } \\
\text { e } \\
\text { informativas } \\
\text { a } \\
\text { comerciantes } \\
\text { y transeúntes } \\
\text { del paseo } \\
\text { ciencias. }\end{array}$} & $\begin{array}{l}\text { Conservatori } \\
\text { o a } \\
\text { transeúntes }\end{array}$ & \multirow[t]{2}{*}{ Humano } & Transeúntes & $\begin{array}{l}\text { Se le dio la } \\
\text { charla a } 25 \\
\text { vendedores }\end{array}$ & $\begin{array}{l}\text { Registro } \\
\text { fotográfico }\end{array}$ \\
\hline & & $\begin{array}{l}\text { Conservatori } \\
\text { o a } \\
\text { vendedores } \\
\text { del área }\end{array}$ & & Vendedores & $\begin{array}{l}\text { Se le dio la } \\
\text { charla a } 15 \\
\text { transeúntes }\end{array}$ & $\begin{array}{l}\text { Registro en } \\
\text { vídeo }\end{array}$ \\
\hline
\end{tabular}




\begin{tabular}{|c|c|c|c|c|c|}
\hline نِّ & $\begin{array}{l}\text { Evaluar la } \\
\text { eficacia de la } \\
\text { propuesta } \\
\text { mediante } \\
\text { observación } \\
\text { directa del } \\
\text { proceso. }\end{array}$ & $\begin{array}{l}\text { Análisis y } \\
\text { descripción } \\
\text { del proceso } \\
\text { de aplicación } \\
\text { de la } \\
\text { propuesta }\end{array}$ & $\begin{array}{l}\text { Libreta de } \\
\text { campo }\end{array}$ & $\begin{array}{l}\text { Se presenta } \\
\text { de manera } \\
\text { descriptiva } \\
\text { el antes, } \\
\text { durante y } \\
\text { después de } \\
\text { la } \\
\text { propuesta. }\end{array}$ & $\begin{array}{l}\text { Documento } \\
\text { trascrito de la } \\
\text { evaluación } \\
\text { de la } \\
\text { propuesta. }\end{array}$ \\
\hline
\end{tabular}

Fuente: Elaboración propia

Para el cumplimiento del primer objetivo se realizó un recorrido por el paseo ciencias y se repartieron 20 dípticos a transeúntes escogidos al azar. Para los vendedores se les entrego en sus locales un total de 20 dípticos. En el contenido de los dípticos se tocan los puntos de las causas, consecuencias y fuentes de los contaminantes en cuestión.

La segunda actividad se realizó al día siguiente y se les dio la charla a 25 vendedores informales del área de estudio, y a 15 transeúntes que accedieron a escuchar el tema. El desarrollo de la misma fue de casi 10 minutos por persona.

Una vez finalizada la etapa de ejecución de la propuesta se reflexiona que la misma tiene su grado de eficacia y que de ser aplicada con una mayor muestra se puede mejorar los aspectos ambientales del área de estudio logrando aminorar las acciones contaminantes de los transeúntes y vendedores. Se hizo difícil poder reunir a todos los vendedores pues por la naturaleza de su trabajo no es viable una reunión grupal, además que no aceptaron. Por lo cual un trato individual y relativamente corto fue más favorable para el cumplimiento del objetivo planteado. En cuanto a los dípticos, estos generaron un impacto favorable pues los transeúntes afirmaron que el diseño de los mismos era didáctico y entendible, por lo cual se espera cierto grado de aprendizaje significativo en la muestra. 
Estrategias para la divulgación de la contaminación sónica y visual en el Paseo Ciencias de Maracaibo, estado Zulia

\section{Conclusiones}

El paseo ciencias es una zona altamente contaminada por elementos sónicos y visuales. La contaminación sónica se encuentra caracterizada por que se percibe a mayor decibelios en los días laborables y en los días de fiestas tales como la feria de la chinita, diciembre y día del niño. Además, prevalece la originada por fuentes fijas; en este caso se encontró las provenientes a equipos de sonido con volumen demasiado altos, ruido de electrodomésticos y los gritos de los vendedores del área. Igualmente, se notó un encubrimiento de algunos ruidos. Las fuentes de contaminación móviles está representada por los medios de transporte terrestre, en menor grado los sistemas de aceleración, bocinas, sirenas de ambulancias, equipos de sonidos de los autobuses y los gritos de los colectores.

Esta abundancia de contaminación por ruidos repercute negativamente en la salud de las personas y en su relaciones con los demás. Se concluye que los efectos a los que están expuestos todos los días las personas que visitan el paseo ciencias son: cefaleas, estrés, fatigas, cansancio, problemas digestivos, mal humor, violencia, irritabilidad, descontento, ofensas, problemas de audición, peleas y discordias.

Examinando las fuentes de contaminación visual presentes en el área se conoció que estas están representadas por vallas publicitarias, basurales improvisados, toldos y locales de vendedores informales, cables, postes, antenas, pintadas y falta de señalizaciones. Ante tal cantidad de contaminación visual las personas expresan los pesares que experimentan, mencionando estrés, cefaleas, repulsión, indignación, depresión y cansancio. También se nombraron las consecuencias que mencionan otros autores entre las cuales están: accidentes automovilísticos, falta de atención, pérdida de valores escénicos, irresponsabilidad y desánimos.

Indagando un poco las bases legales correspondientes a la publicidad exterior en Venezuela y a la contaminación sónica (República Bolivariana de Venezuela, 1992, Congreso de la república de Venezuela, 1976, Ministerio del Poder Popular para las 


\section{César Morales}

Telos Vol. 21, No. 2 (2019). 347-371

Obras Públicas y Vivienda, 2007), se encontró que los entes encargados no están haciendo nada por cumplir las leyes, reglamentos y decretos relacionados a estos contaminantes. Es decir que hay una falta de aplicación de las normativas legales en cuanto a la colocación de vallas publicitarias y exceso de las mismas, así como la música con volúmenes exagerados que perjudican la salud de los habitantes.

Finalmente, se encontró que las personas al momento de participar en algún proyecto o programa de recuperación del paseo ciencias tienen una actitud sumamente pasiva. Esto es generado por la misma contaminación visual y por la falta de sentido de pertenencia con respecto al área de estudio.

\section{Referencias Bibliográficas.}

Alcaldía del Municipio Maracaibo (1992). Sector Santa Lucía patrimonio histórico arquitectónico, Decreto No.37 del 08 de septiembre. Venezuela.

Alfie, Miriam y Salinas, Osvaldo (2017). Ruido en la ciudad. Contaminación auditiva y ciudad caminable. Estudios demográficos y urbanos. Volumen. 32, No. 1. México (Pp. 65-96).

Asamblea Legislativa del Estado Zulia (1976). Remodélese y consérvese al barrio Santa

Lucia. Área especialmente afectada de expropiación por Causa de Utilidad Pública o Social. Gaceta Oficial Número 81-A, 3.736 del 06 de octubre. Venezuela.

Asamblea Nacional de la República Bolivariana de Venezuela (2013). Zona de Interés Turístico al Casco Central de Maracaibo, Decreto No.373 Gaceta Oficial N ${ }^{\circ}$ 40.246 del 09 de Septiembre. Venezuela.

Ayala, Jessica (2017). Características y efectos de la contaminación visual de carácter publicitario en la ciudad de asunción. Paraguay. Tesis maestría. Magister Scientiae en Manejo de Recursos Naturales y Gestión Ambiental del Territorio. Facultad de Ciencias Agrarias, Universidad Nacional de Asunción. Paraguay.

Borda, Pablo. Dabenigno, Valeria. Freidin, Betina y Güelman Martín (2017). Estrategias para el análisis de datos cualitativos. Desarrollo Editorial Carolina De Volder - Centro de Documentación e Información, IIGG. Argentina.

Camacaro, Leriz y López, Mapy (2015). Potencialidades de Santa Lucía -Maracaibo para la implementación del servicio de Posadas Turísticas, bajo el enfoque del 
Estrategias para la divulgación de la contaminación sónica y visual en el Paseo Ciencias de Maracaibo, estado Zulia

Cuadro de Mando Integral. Portafolio. Volumen 1, No. 31.Venezuela (Pp. 3240).

Canelones, Marín (2007). Contaminación sónica en la zona residencial del casco

central de Maracaibo. Trabajo especial de grado. Especialización en

Educación Ambiental. Universidad Rafael Urdaneta. Venezuela.

Congreso de la República de Venezuela (1994). Santa Lucia "área de valor histórico".

Gaceta Oficial Número 35.441 del 15 de abril. Venezuela.

Congreso de la república de Venezuela (1976). Ley Orgánica del Ambiente. Gaceta número 31.004. Venezuela.

Contraloría General de la República (2016). Actuaciones -Anexo al Informe de Gestión 2016. Venezuela.

Espejo, Washington (2014). Contaminación visual y propuesta de regulación en el distrito de Wanchaq - Cusco. Tesis de grado. Universidad Nacional de San Antonio Abad del Cusco. Perú.

Fuentes, Valeria y Arguello, Anita (2015). Indicadores de contaminación visual y sus efectos en la población. Enfoque UTE. Volumen 6, No. 3. Ecuador (Pp. 115132).

González, Yamile y Fernández, Yaìna (2014). Efectos de la contaminación sónica sobre la salud de estudiantes y docentes, en centros escolares. Revista Cubana de Higiene y Epidemiología. Volumen 52, No. 3. Cuba (Pp. 402-410).

Guevara, María. Téllez, María y Flores, María (2015). Aprovechamiento sustentable de los recursos naturales desde la visión de las comunidades indígenas: Sierra Norte del Estado de Puebla. Nova Scientia. Volumen 7, No. 14. México (Pp. 511-537).

Guijarro, Joshelline. Terán, Ivanna e Valdez, Mercedes (2015). Determinación de la contaminación acústica de fuentes fijas y móviles en la vía a Zamborondón en Ecuador. Ambiente y Desarrollo. Volumen 20, No. 38. Colombia (Pp. 4151).

Méndez, Carmen (2013). La contaminación visual de espacios públicos en Venezuela.

Gestión y Ambiente. Volumen 16. No. 1. Colombia (Pp. 45-60).

Morales, César (2016). Exporeciclaje 2015. Estrategia de educación ambiental.

Educ@ción en Contexto. Volumen 2, No. Especial. Venezuela (Pp. 72-83).

Mosqueda, Gradys (2012). Diseño de un manual de estrategias para el mejoramiento del desempeño del acompañante pedagógico en el centro de educación inicial Bicentenario de valle de la pascua, estado Guárico. Tesis 
maestría en educación inicial. Universidad Latinoamericana del caribe. Venezuela.

Muñoz, Desiree (2012). Impacto de la contaminación sonora sobre la estructura del suelo urbano caso: Parroquia Juana de Ávila. Tesis de maestría en catastro y evaluó inmobiliario urbano. Universidad del Zulia. Venezuela.

Ministerio del Poder Popular para las Obras Públicas y Vivienda (2007). Resolución 046 sobre publicidad móvil y fija. Gaceta número 38.750. Venezuela.

Miyara, Federico y Sanguineti, Jorge (1996). La contaminación acústica en los medios de transporte urbano de rosario. 20 Encuentro Interdisciplinario de Trabajo y Salud, UNR. Rosario. Argentina.

República Bolivariana de Venezuela (1992). Decreto 2217 sobre el control de la contaminación generada por ruido. Gaceta oficial número 4418. Venezuela.

Rosales, Rosaura (2014). Estrés y salud. Tesis de grado. Universidad de Jaén. España. Sáez, María (2011). Contaminación visual por antenas de televisión en el Albaicín. Una propuesta tecnológica para su corrección. Revista electrónica de patrimonio histórico. No. 9. España (Pp. 1-30). 